\title{
Studies on the Extraction of Soft Acid Metal Species Using MIDOA and Analogous Compounds
}

\author{
Yuji SASAKI, ${ }^{1}$ Morihisa SAEKI, ${ }^{1}$ Yumi SUGO,,${ }^{1}$ Yasuhisa IKEDA, ${ }^{2}$ Takeshi KAWASAKI, ${ }^{2}$ \\ Tomoya SUZUKI ${ }^{2}$ and Akira OHASHI ${ }^{3}$ \\ ${ }^{1}$ Japan Atomic Energy Agency, Tokai, Ibaraki 319-1195, Japan \\ ${ }^{2}$ Tokyo Institute of Technology, 2-12-1-N1-34 Ookayama, Meguro-Ku, Tokyo 152-8550, Japan \\ ${ }^{3}$ Ibaraki University, 2-1-1 Bunkyo, Mito, Ibaraki 310-8512, Japan \\ (Received March 24, 2014; Accepted May 20, 2014)
}

The extractant, methylimino-bis- $N, N^{\prime}$-dioctylacetamide (MIDOA), was used for the extraction of soft acid metals. It was found that MIDOA can extract not only $\mathrm{Cr}(\mathrm{VI}), \mathrm{Mo}(\mathrm{VI}), \mathrm{W}(\mathrm{VI}), \mathrm{Tc}(\mathrm{VII})$ and $\operatorname{Re}(\mathrm{VII})$, whose metals can form oxonium anions due to their high oxidation states, but also other metal cations, like $\mathrm{Nb}(\mathrm{V}), \mathrm{Ta}(\mathrm{V})$ and $\mathrm{Pd}(\mathrm{II}) . \quad$ Analogous compounds, imino-bis- $N, N^{\prime}$-dioctylacetamide (IDOA) and methylimino-bis- $N, N$ '-di-2-ethylhexylacetamide (MIDEHA), were synthesized and compared for their extractability. It is clear that these extractants have almost the same or lower $D$ values than those for MIDOA. In order to examine the effect on extractability with different donor atoms, TODGA $\left(N, N, N^{\prime}, N^{\prime}\right.$-tetraoctyl-diglycolamide) and TDGA $\left(N, N, N^{\prime}, N^{\prime}\right.$ '-tetraoctyl-tyiodiglycolamide) having oxygen and sulfur donor were employed. The comparison of their extractabilities suggests that the trend of $\mathrm{Pd}$ and Re extraction is $\mathrm{N}>\mathrm{S}>\mathrm{O}$ and $\mathrm{N}>\mathrm{O}>\mathrm{S}$, respectively. The chemical stability and the structure of MIDOA were also studied and discussed in this paper.

\section{Introduction}

Although several extractants, like diglycolamides, malonamides and carbamoylmethyl phosphine oxides, have been developed for the recovery of actinides[1-6], information on novel extractants to extract the platinum metal group and oxonium ions is rarely reported. The reason why there are few reports is due to the difficulty in their complex formation, inert metal ions, and negative charges after formation of the oxonium ions. Recently, a few N-donor extractants have been developed and confirmed to have high distribution ratio $(D)$ values for $\mathrm{Pd}(\mathrm{II})$ and $\mathrm{Tc}(\mathrm{VII})$ [7-8]. These metals are soft-acid metals and are expected to react strongly with soft donor atoms, based on the HSAB principle[9]. In the atomic energy field the separation of $\mathrm{Pd}, \mathrm{Ru}, \mathrm{Rh}, \mathrm{Mo}$ and Tc from high level radioactive waste is important to reduce the environmental risk, and a four-group partitioning process has been developed in Japan [10-11].

The extractant, MIDOA (methylimino-bis- $N, N^{\prime}$-dioctylacetamide) used mainly here was developed in our group and reported on in 2007 for the first time [12-13]. This compound belongs to the podand-type, which has a structure similar to diglycolamide, with a nitrogen atom introduced into the carbon chain connected the two amide groups. Therefore, MIDOA has a high reactivity with soft-acid metals and shows high $D$ values for Tc(VII) and Pd(II). In this work, the extractability, stability and the chemical structure of metal-complexes have been investigated. In addition, analogous compounds to MIDOA and 
extarctants having oxygen and sulfur atoms are employed for comparison of their extractabilities. General structures used here are shown in Figure 1. These are imino-bis- $N, N^{\prime}$-dioctylacetamide (IDOA), methylimino-bis- $N, N^{\prime}$-di-2-ethylhexylacetamide (MIDEHA), $N, N, N^{\prime}, N^{\prime}$-tetraoctyldiglycolamide (TODGA), $N, N, N$ ', $N$ '-tetraoctyl-thiodiglycolamide (TDGA) and methylimino-bis- $N, N$ '-diethylacetamide (MIDEA). MIDOA, IDOA, MIDEHA, TODGA and TDGA can be readily dissolved in $n$-dodecane, for use in solvent extraction. Furthermore, MIDEA is soluble in water and is used for the preparation of metal complexes.

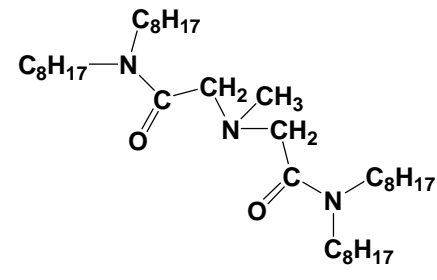

MIDOA<smiles>CCCCCCCN(CCCC)C(=O)COCC(=O)N(CCCC)CCCC</smiles>

TODGA<smiles>CCCCCN(CCC)C(=O)CNCC(=O)N(CCC)CCCC</smiles>

IDOA

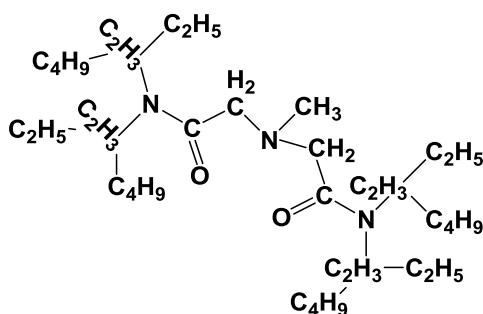

MIDEHA<smiles>CCCCCN(CCCC)C(=O)CSCC(=O)N(CCCC)CCCC</smiles>

$\operatorname{TDGA}(\mathrm{C} 8)$<smiles>CCCCCCN(CC)C(=O)CN(C)CC(=O)N(CC)CC</smiles>

MIDEA

Figure 1. Structures of extractants used in this work.

\section{Experimental}

\subsection{Syntheses of extractants and metal-organic compounds}

The syntheses of MIDOA and its analogous compounds, described in previously reports [7, 12], were carried out using the amide synthetic method using a carboxylic acid and an amine. MIDOA, so obtained, was purified by silica-gel chromatography.

The synthetic method for production of the precipitate of the MIDEA-Re complex is described below. Solvent extraction was performed using an organic phase consisting of MIDEA dissolved in dichloromethane and Re in $0.2 \mathrm{M} \mathrm{HNO}_{3}$. After mixing with both phases, the organic phase was taken and evaporated gently at room temperature overnight. The white precipitate, which formed in the concentrated organic phase, was recovered. The sample was washed with a small volume of $n$-hexane, dried and kept in the refrigerator.

\subsection{The solvent extraction method}

Solvent extraction experiments were carried out using the following method. The extractant was dissolved in $n$-dodecane without heating and used as the organic phase, and $\mathrm{HNO}_{3}$ containing a certain metal was used as the aqueous phase. One milliliter of the organic phase was mixed with the same volume of the aqueous phase. The mixture was shaken mechanically at $1000 \mathrm{rpm}$ for $30 \mathrm{~min}$ at $25 \pm 0.1$ ${ }^{\circ} \mathrm{C}$. After centrifugation, the concentrations of metal ions in the aqueous phase were measured using 
ICP-AES (SPS 3100, Seiko Instruments Inc.) or ICP-MS (SPQ 9000, Seiko-EG\&G). The metal concentrations in the organic phase were obtained from the difference between the initial and equilibrium aqueous phase concentrations. The distribution ratios $(D)$ were obtained from the ratio of the metal concentration in the organic phase to that in the aqueous phase $\left([\text { metal }]_{\text {org }} /[\text { metal }]_{\mathrm{aq}}\right)$.

\subsection{Structural study}

A sample of the Re-MIDEA complex was located on a glass fiber and cooled by a refrigeration apparatus having an air-blowing system. Then, the structure of the sample was measured by X-ray diffractometer (Rigaku RAXIS Rapid). The ${ }^{1} \mathrm{H}$-NMR signals for MIDOA before and after extraction of Re were measured using a spectrometer (Bruker Avance III $400 \mathrm{MHz}$ ). The chemical shifts between the two samples, standardized by tetramethyl-silane, were obtained. The evaluation of the optimized structures was checked by harmonic frequency analysis [14]. Most of the computations were carried out on Fujitsu PrimeQuest and Hitachi SR16000 computers.

\subsection{Evaluation of chemical and physical properties of reagents}

The dissociation of MIDEA was studied by the potentiometric method. MIDEA dissolved in the aqueous phase was titrated with acid and the $\mathrm{pH}$ values were measured by a $\mathrm{pH}$ meter (Hiranuma, COM-1500). The physical properties of MIDEA and its metal-complexes were studied at high temperature. The sample weight and heat generation are recorded with temperature up to $500{ }^{\circ} \mathrm{C}$ using thermogravimetry (Rigaku, Thermo plus EVO II). The chemical and physical stabilities (hydrolysis and radiolysis) were studied by solvent extraction using the organic phase before and after mixing with acid and irradiation by gamma rays. The relation of the $D(\mathrm{M})$ values with mixing time and the absorbed dose in gamma irradiation were investigated. Here, gamma irradiation was carried out at the cobalt-60 irradiation facility in the Takasaki Advanced Radiation Research Institute, Japan Atomic Energy Agency (JAEA).

\section{Results and Discussion}

\subsection{Extractions of metal ions by MIDOA}

The extractions of the metal ion $(\mathrm{Cr}(\mathrm{VI}), \mathrm{Mo}(\mathrm{VI}), \mathrm{W}(\mathrm{VI}), \mathrm{Nb}(\mathrm{V}), \mathrm{Ta}(\mathrm{V}), \mathrm{Tc}(\mathrm{VII}), \operatorname{Re}(\mathrm{VII})$ and $\mathrm{Pd}(\mathrm{II}))$ by MIDOA are shown in Figure 2. As a soft donor, N, included in the center of alkyl chain backbone, MIDOA favors the soft acid metals described above, especially $\mathrm{Tc}(\mathrm{VII}), \operatorname{Re}(\mathrm{VII})$ and $\operatorname{Pd}(\mathrm{II})$. The extraction behavior with respect to $\mathrm{HNO}_{3}$ and the extractant concentrations are shown in Figure 2(a) and Figure 2(b), respectively. Here, the molar concentration, $\mathrm{mol} / \mathrm{dm}^{3}$, is defined as $\mathrm{M}$ in the following sentences.

A gradual decrease in $D$ with $\mathrm{HNO}_{3}$ concentration can be seen in Figure 2(a). Due to the competition reaction with $\mathrm{NO}_{3}{ }^{-}$, this behavior is peculiar to anion extractions [15-16]. $\mathrm{Nb}(\mathrm{V})$ and $\mathrm{Ta}(\mathrm{V})$ show different behavior from the other metals, and a gradual increase of $D$ with $\mathrm{HNO}_{3}$ can be seen from around $1 \mathrm{M}$. Although the decrease and the increase in the $D(\mathrm{M})$ values can be explained by the protonation of MIDOA and the ion pair extraction with $\mathrm{NO}_{3}^{-}$, there is not enough information to allow discussion of the extraction behavior of $\mathrm{Nb}$ and $\mathrm{Ta}$.

Figure 2(b) shows the linear relationships between $\log$ [extractant] vs. $\log D$. The nature of the chemical species extracted in the organic phase can be determined. The slope values are as follows; $\mathrm{Cr}$ : 0.49, Nb; 0.85, Ta; 0.84, Mo; 1.17, W; 0.37, Tc; 1.06, Re; 0.84, and Pd; 1.61. These slope values suggest 
that the molar ratio of these metal extraction species are mainly 1:1, except for Pd.
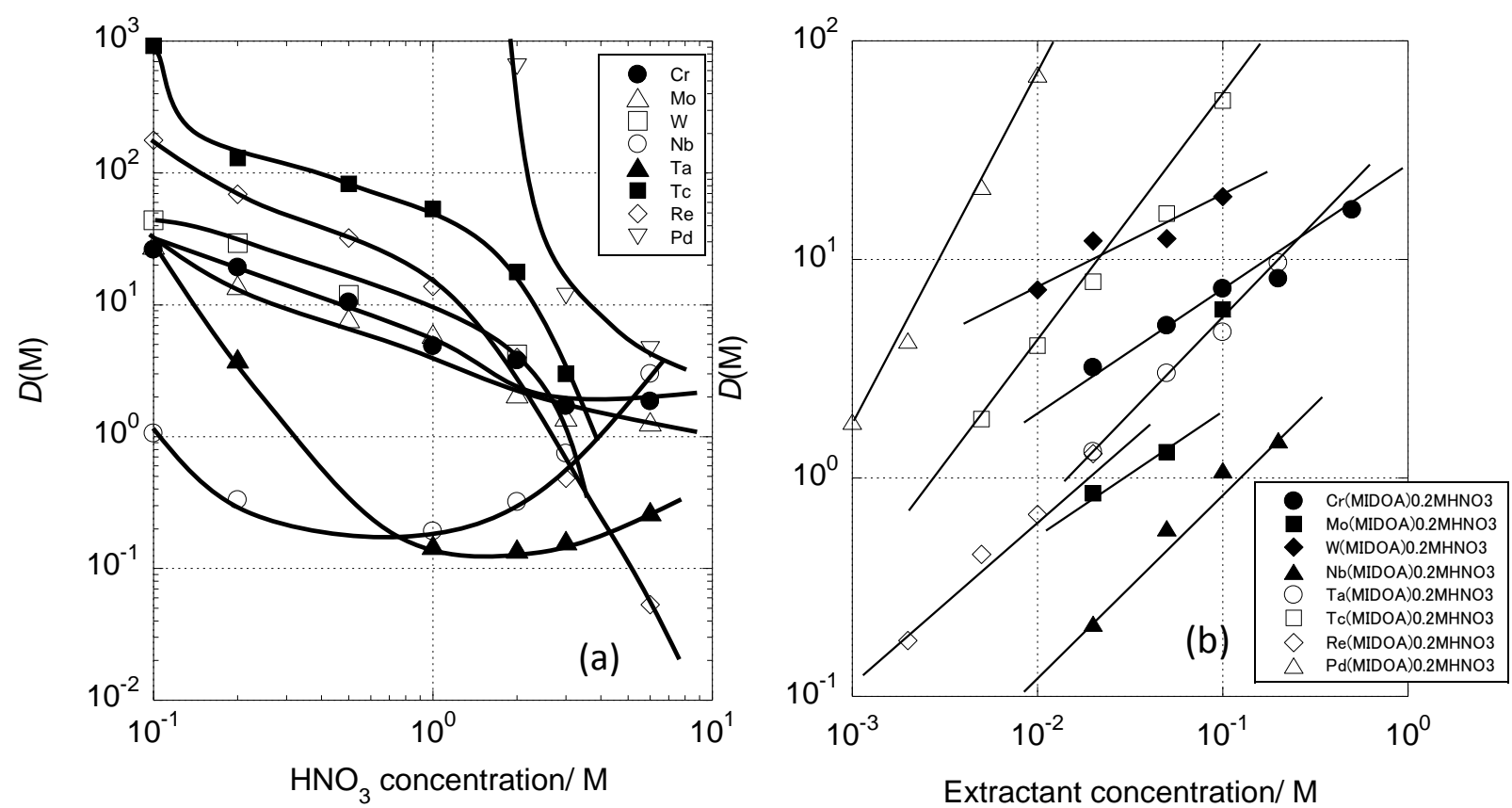

Figure 2. $\mathrm{Cr}(\mathrm{VI}), \mathrm{Mo}(\mathrm{VI}), \mathrm{W}(\mathrm{VI}), \mathrm{Nb}(\mathrm{V}), \mathrm{Ta}(\mathrm{V}), \mathrm{Tc}(\mathrm{VII}), \operatorname{Re}(\mathrm{VII})$ and $\mathrm{Pd}(\mathrm{II})$ extraction by MIDOA.

(a): Dependence of $D(\mathrm{M})$ on the $\mathrm{HNO}_{3}$ concentration for $0.1 \mathrm{M} \mathrm{MIDOA} / n$-dodecane.

(b): Dependence of $D(\mathrm{M})$ on the MIDOA concentration at $0.2 \mathrm{M} \mathrm{HNO}_{3}$.

\subsection{Comparison of extraction performance of MIDOA with other analogous compounds}

The extractants with the same central frame to MIDOA but with different attachments of alkyl groups were studied and their extraction performance compared. IDOA and MIDEHA in Figure 1 as well as MIDOA were employed in this work. Here, the characteristics dependent on their structures are summarized; IDOA has a hydrogen atom attached in the center of the $\mathrm{N}$ donor atom, which can form a hydrogen bond with the $\mathrm{O}$ atom in the molecule, thus lowering the $D$ values. MIDEHA has 2-ethylhexyl alkyl groups of attached to amidic $\mathrm{N}$ donors, which will cause steric hindrance, thus causing the $D$ values to decrease. Figure 3 shows the relationship between $D(\mathrm{Pd}, \mathrm{Re})$ and the concentration of these extractants. Higher $D(\mathrm{Pd})$ values for MIDOA than those for IDOA and MIDEHA can be seen in Figure 3, however, the $D(\mathrm{Re})$ values for the three extractants are almost the same. These results suggest that the intermolecular hydrogen bond for IDOA and the steric hindrance for MIDEHA are uncertain in the case of Re extraction.

Solvent extraction with the different donor atoms was also investigated. In this work, $\mathrm{N}$ donor MIDOA, O donor TODGA and S donor TDGA are compared for the extraction of Pd(II) and Re(VII). Figure 4 shows the relationship between $D(\mathrm{Pd}, \mathrm{Re})$ and the concentration of these extractants. There is a certain difference among the extractabilities for the three extractants, the trend of the $D$ values are MIDOA > TDGA > TODGA for Pd and MIDOA > TODGA > TDGA for Re. MIDOA has the highest $D$ values for both metal ions. Here, Pd(II) belongs to the soft acid metal group and $\operatorname{Re}(\mathrm{VII})$ forms an oxonium anion with four oxygen atoms, so these metals are likely to favor soft donor atoms. As is well-known, TODGA 
has a strong extractability for lanthanides and actinides, which are hard acid metals [5-6, 8]. It appears that the sulfur donor atom does not strongly complex with $\operatorname{Re}(\mathrm{VII})$.

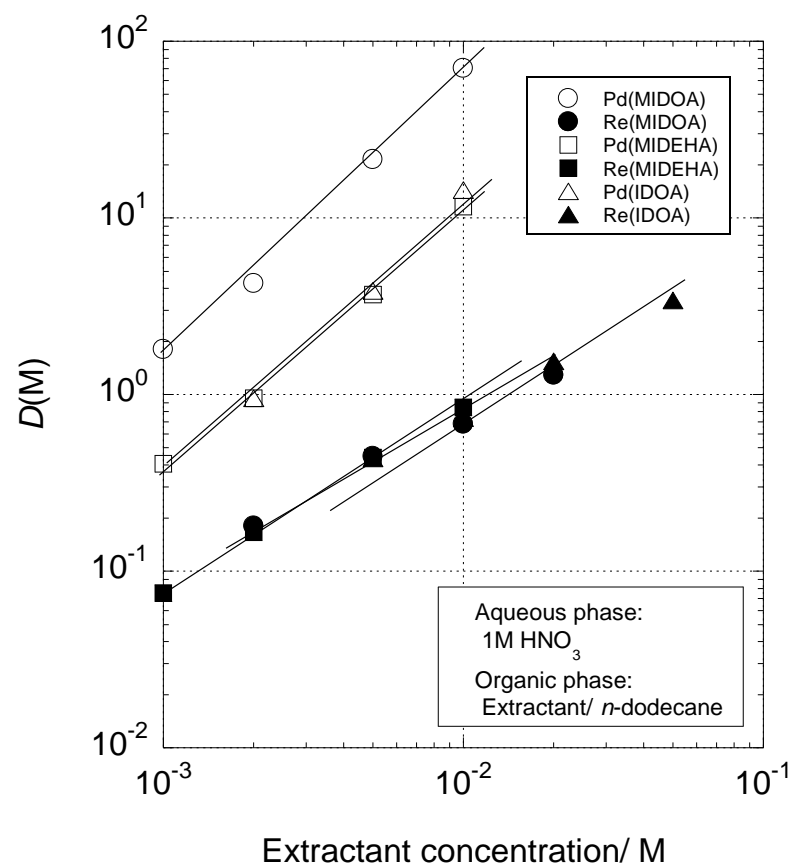

Figure 3. Comparison of $D(\mathrm{Pd}, \mathrm{Re})$ with MIDOA, MIDEHA and IDOA.

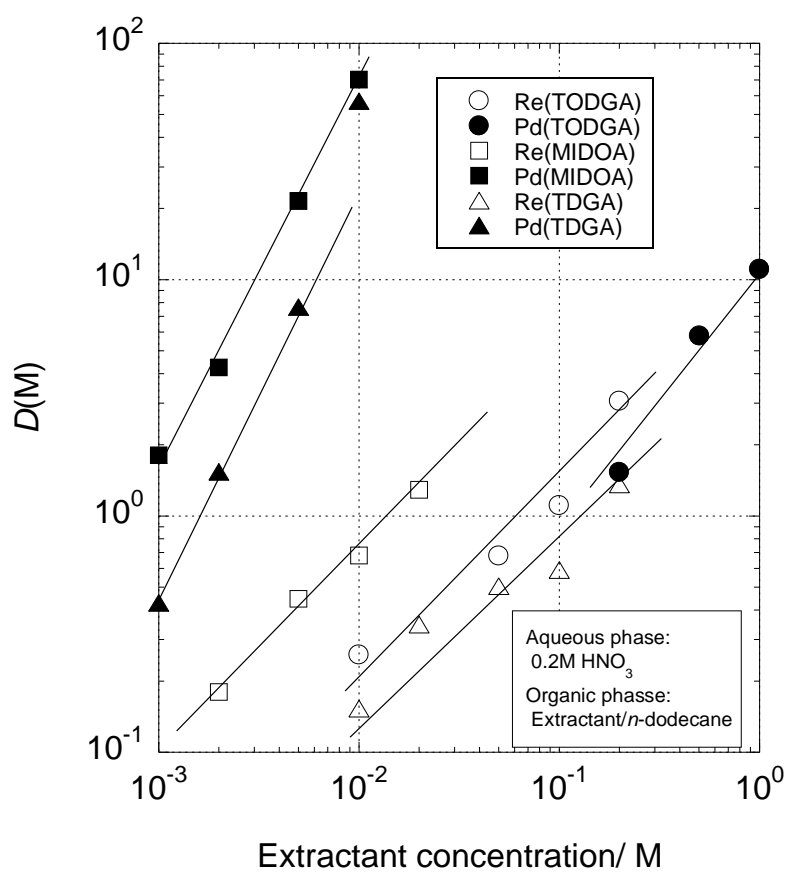

Figure 4. Comparison of $D(\mathrm{Pd}, \mathrm{Re})$ with MIDOA, TODGA and TDGA.

\subsection{Stability and physical properties of MIDOA and MIDEA}

The dissociation constant of MIDEA obtained in this work is shown in Table 1, together with those for some other secondary amines. The $p \mathrm{~K}$ value for MIDEA, 6.919, is relatively small in comparison with the other amines, which suggests that the tertiary amine and amide are less protonation than the secondary amines.

Table 1 Dissociation constants of MIDEA and other secondary amines.

\begin{tabular}{lc}
\hline & $p K$ \\
MIDEA & 6.919 \\
& \\
Dimethylamine & $10.77^{*}$ \\
Diethylamine & $10.93^{*}$ \\
Diethanolamine & $8.9^{*}$ \\
Diethylenetriamine & $4.23\left(p K_{1}\right), 9.02\left(p K_{2}\right), 9.84\left(p K_{3}\right)^{*}$ \\
\hline
\end{tabular}

*: Data are obtained from the reference[17]. 
The hydrolytic and radiolytic stability of MIDOA was studied. After stirring with $\mathrm{HNO}_{3}$ or irradiation with gamma-rays, the organic solvents were ready for use in solvent extraction. The $D$ values are plotted against mixing time (Figure 5(a)) and the dose of gamma-radiation (Figure 5(b)). In these figures, the results for IDOA, TODGA (or TDdDGA, $N, N, N^{\prime}, N^{\prime}$-tetradodecyldiglycolamide) exposed to the same conditions are also shown. All of the $D$ values decrease with mixing time and absorbed dose, which indicates that the extractant concentration in the organic phase is decreased by decomposition with acid and gamma-irradiation. As shown in these figures, the ratio of decreasing $D$ with mixing time and dose is higher for DGA than MIDOA and IDOA, which indicates that the N-donor extractants, MIDOA and IDOA, have a somewhat higher stability with regard to acid and radiation than DGA.
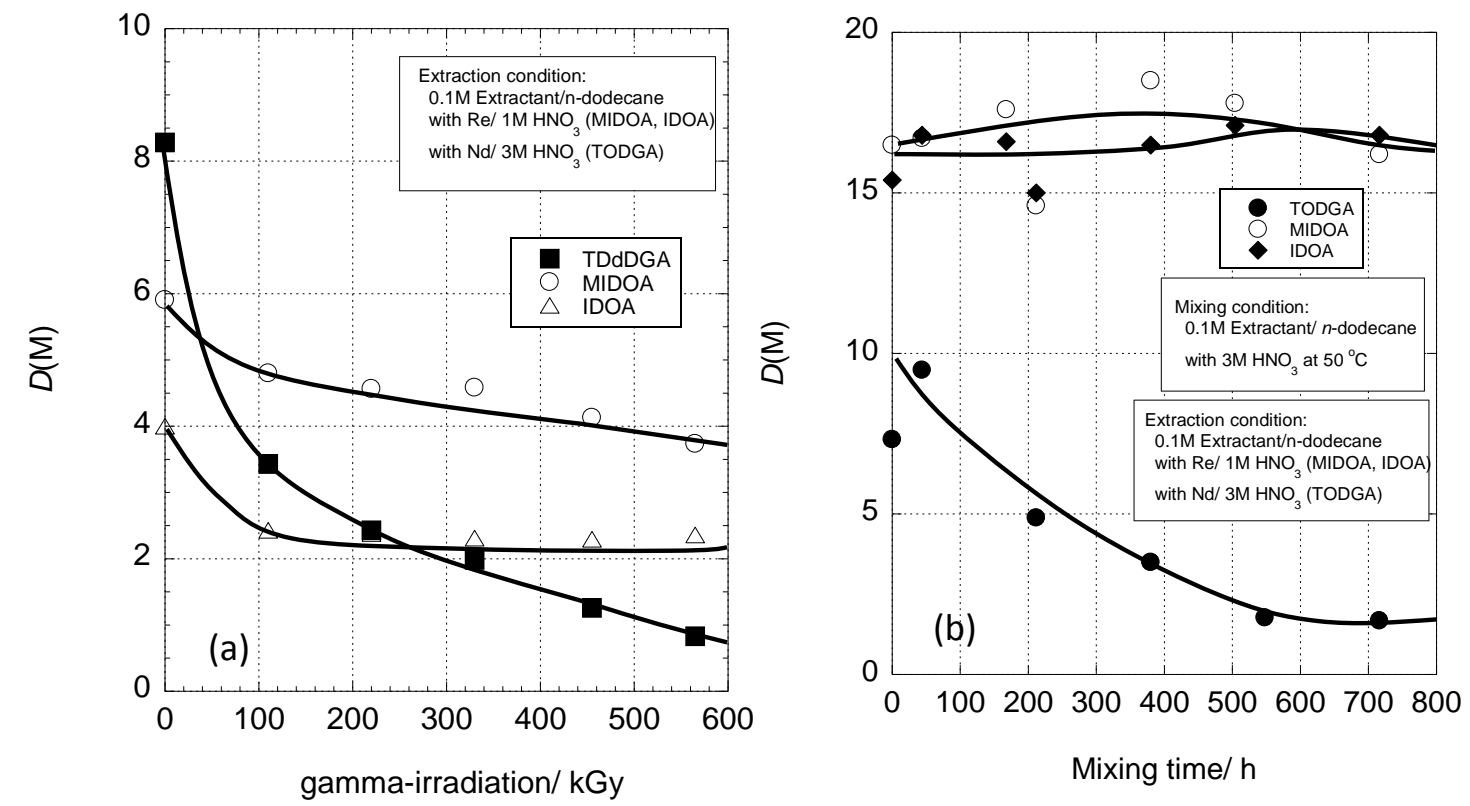

Figure 5. Variation of $D$ values using MIDOA, IDOA and DGA as the extraction solvent with respect to gamma irradiation and mixing time with acid.

(a) Dependence on gamma irradiation, (b) Dependence on mixing time with acid.

\subsection{Chemical structure of MIDEA-Re and the MIDOA-Re complex}

Figure 6 is a photograph of the Re-MIDEA complex, a white precipitate, obtained by the procedure described in the experimental section. In order to confirm the successful preparation of the MIDEA-Re complex, the sample was characterized by thermo gravimetric analysis and differential thermal analysis and compared to that of MIDEA itself. Figure 7 shows the temperature, weight and heat generation against operation time for the samples of MIDEA(a) and MIDEA-Re(b). Initially MIDEA is a liquid at room temperature, its weight decreases with an increase in temperature. Less heat generation were recorded in Figure 7(a), so the sample might be evaporated at about $150{ }^{\circ} \mathrm{C}$.

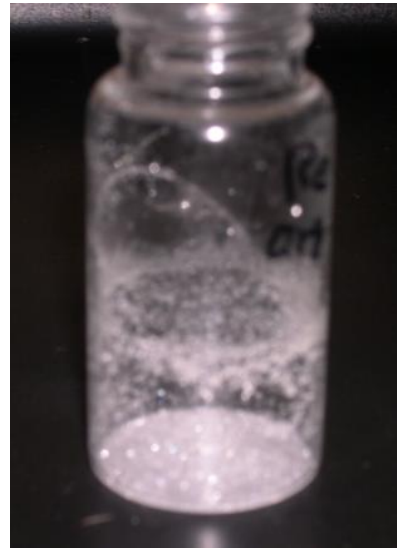

Figure 6. White precipitate of the Re-MIDEA complex. 
Figure 7(b) shows the results for the Re-MIDEA complex. Here, the precipitate is subjected to elemental analysis, the results of which indicate a molar ratio of 1.2:1 for Re:MIDEA, The metal-complex is a solid at room temperature. As the temperature increases, an endothermic reaction at $208{ }^{\circ} \mathrm{C}$ can be seen, which is due to the phase transition to liquid. After that, the weight of the sample decreases with strong heat generation, which indicates that the sample is decomposed by heat. Throughout the thermo gravimetric and thermal analysis studies, two samples, MIDEA and the MIDEA-Re complex, show their own characters, which prove the individual substances of samples prepared.

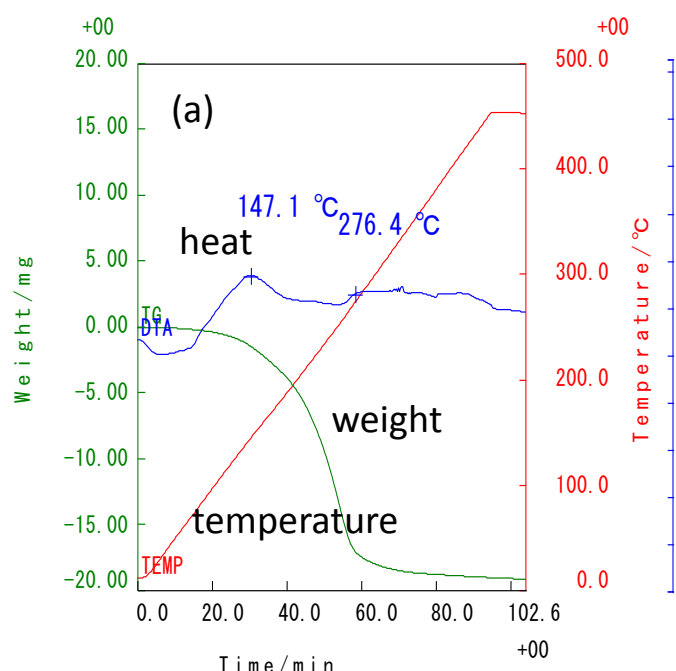

MIDEA

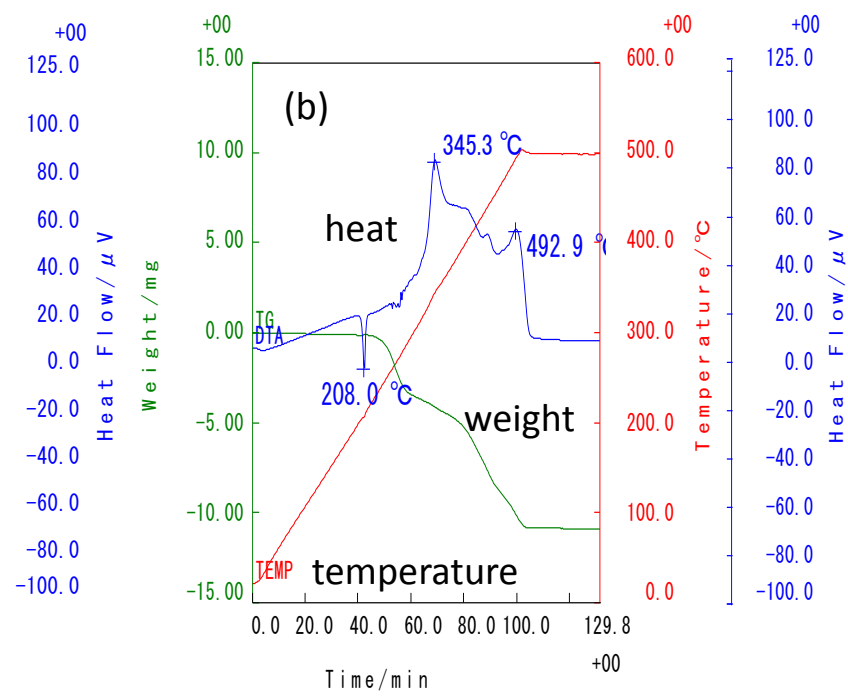

MIDEA-Re

Figure 7. Relationship between temperature, sample weight, and heat generation and operating time for the thermo gravimetric and thermal analysis. (a): MIDEA, (b): MIDEA-Re complex.

Using this sample, the chemical structure is determined by X-ray diffractometry. The results are shown in Figure 8. The bond lengths, Re-O, $\mathrm{O}=\mathrm{C}$, and $\mathrm{N}-\mathrm{C}$, are shown in this figure, however, there is no information on the bond length between Re and N. Because of the long distance, the bond length can hardly be estimated by this method. It seems that there exists a longer Re-N bond than the normal atomic distance, like a hydrogen bond.

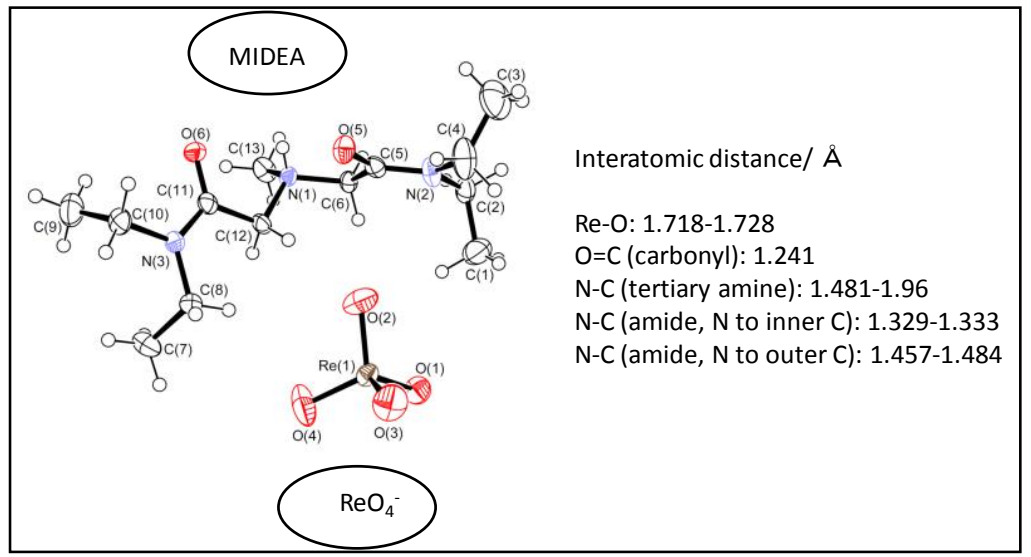

Figure 8. Information on the structure of the Re-MIDEA complex obtained by X-ray diffractometry.

From the results of the X-ray diffraction study, the optimized structure calculated[14] is shown in Figure 9. Three hydrogen bonds exist and connect $\mathrm{H}$ in MIDEA with the $\mathrm{O}$ in the Re oxonium ion. In 
order to confirm this result experimentally, we performed a ${ }^{1} \mathrm{H}-\mathrm{NMR}$ study, especially with regard to the chemical shifts of the samples before and after Re extraction. Figure 10 shows the ${ }^{1} \mathrm{H}-\mathrm{NMR}$ signals of samples of MIDOA itself and the MIDOA-Re complex after extraction. The spectrum for MIDOA is illustrated in the upper part and that of MIDOA-Re is in the lower part of Figure 10. The signals (4) and (5) are clearly shifted to a lower magnetic field ((4); from 3.6 to $4.6 \mathrm{ppm}$, (5); from 2.6 to $3.3 \mathrm{ppm}$ ), on the other hand, signal (3) is less shifted between the two samples (from 3.3 to $3.2 \mathrm{ppm}$ ). The signals (3), (4) and (5) are identified as CO-N-CH${ }_{2}{ }_{-}, \mathrm{N}_{-} \mathrm{CH}_{2}{ }_{-}-\mathrm{CO}$ and $\mathrm{C}-\mathrm{NCH}_{3}{ }^{*} \mathrm{C}$, respectively. Due to the hydrogen bonds with $\mathrm{ReO}_{4}{ }^{-}$as shown in Figure 9, signals (4) and (5) will shift to a lower field before and after Re extraction. This would be evidence of the structure for the Re-MIDOA complex in Figure 9.

\section{${ }^{1} \mathrm{H}-\mathrm{NMR}$ study for MIDOA-Re complex}

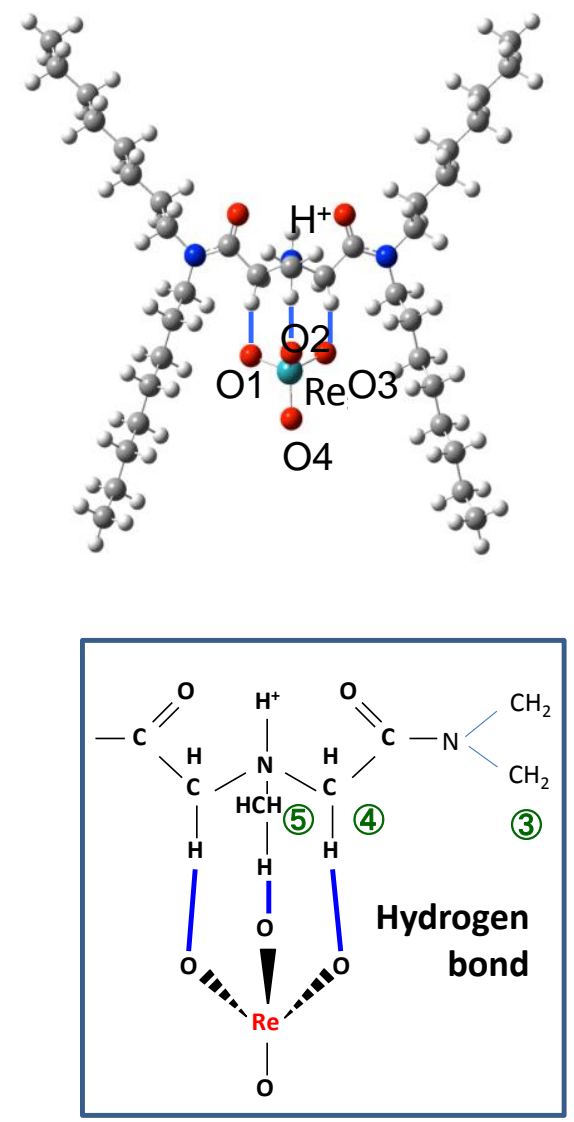

Figure 9. Three hydrogen bonds in the optimized structure of Re-MIDOA.

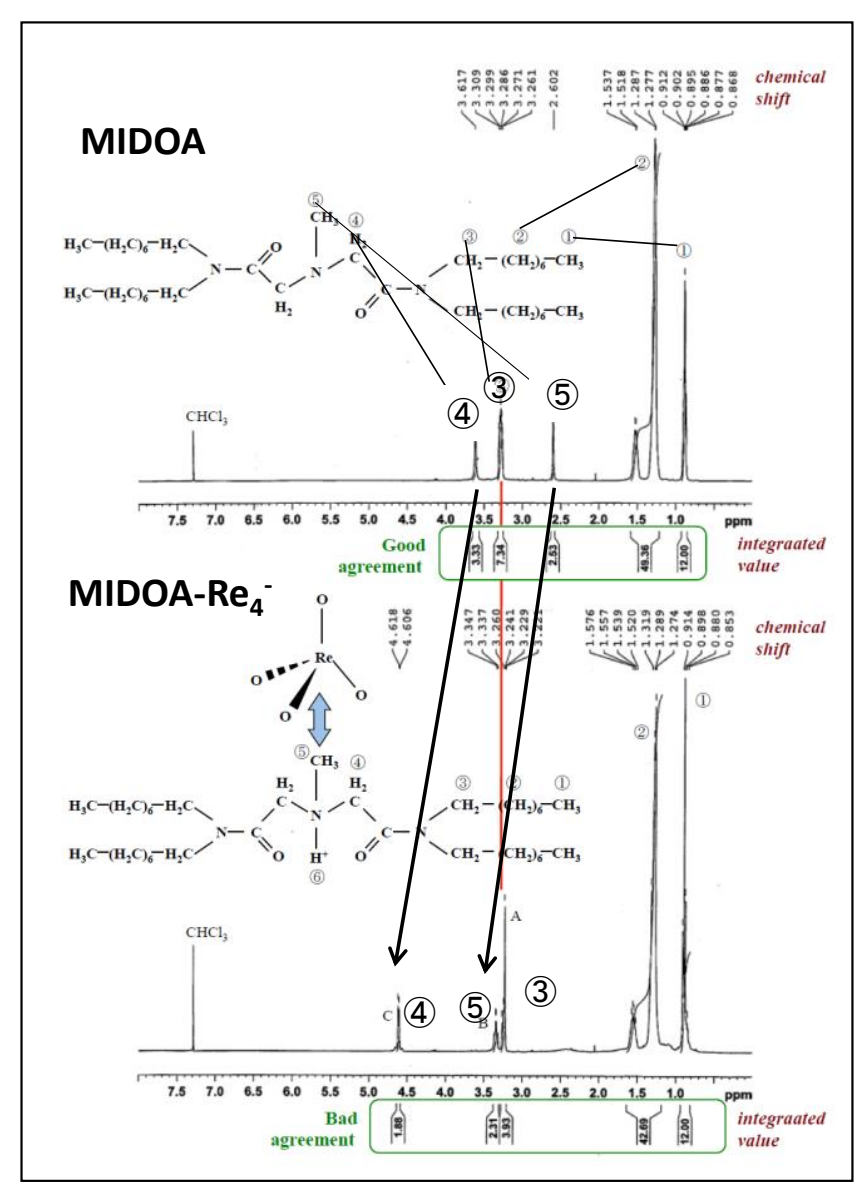

Figure 10. ${ }^{1} \mathrm{H}-\mathrm{NMR}$ study for MIDOA and Re-MIDOA complex.

\section{Conclusion}

A study of the solvent extraction performance, stability, physical properties, and chemical structure using MIDOA and its analogous compounds has been carried out. The results are summarized below: (1) 
MIDOA has a strong extractability, especially, for $\mathrm{Tc}(\mathrm{VII}), \operatorname{Re}(\mathrm{VII})$ and $\mathrm{Pd}(\mathrm{II})$, (2) MIDOA is the best extractant for $\mathrm{Pd}(\mathrm{II})$ among the extractants used here, (3) relatively strong stability for hydrolysis and radiolysis can be seen for MIDOA, and (4) the structural studies show that the hydrogen bonds are related strongly to their complexation.

\section{Acknowledgement}

The authors would like to sincerely thank the staffs of Wako- Pure Chemical Industries for performing organic syntheses, and Mr. Y. Nakahara and the late Mr. M. Ito of JAEA for performing solvent extraction experiments, and Drs. T. Kimura, Y. Morita and K. Morita of JAEA and Dr. M. Nakase and Profs. K. Takeshita and M. Ozawa of Tokyo Inst. Technol. for useful discussions.

This work was partially supported by MEXT, Grant-in-Aid for Scientific Research (A) (21246146).

\section{References}

1) E.P. Horwitz, A.C. Muscatello, D.G. Kalina, L. Kaplan, Sep. Sci. Technol., 16, 417-437 (1981).

2) E.P. Horwitz, D.G. Kalina, H. Diamond, G.F. Vandegrift, Solvent Extr. Ion Exch., 3, 75-109 (1985).

3) G. Thiollet, C. Musikas, Solvent Extr. Ion Exch., 7, 813-827 (1989).

4) C. Cuillerdier, C. Musikas, P. Hoel, L. nigond, X. Vitart, Sep. Sci. Technol., 26, 1229-1244 (1991).

5) Y. Sasaki, Y. Sugo, S. Suzuki, S. Tachimori, Solvent Extr. Ion Exch., 19, 91-103 (2001).

6) Y. Sasaki, Y. Kitatsuji, Y. Sugo, Y. Tsubata, T. Kimura, and Y. Morita, Solvent Extr. Res. Dev. Jpn 19, 51-61 (2012).

7) Y. Sasaki, M. Ozawa, T. Kimura, K. Ohashi, Solvent Extr. Ion Exch., 27, 378-394 (2009).

8) Y. Sasaki, Y. Tsubata, Y. Kitatsuji, Y. Sugo, N. Shirasu, Y. Morita, T. Kimura, Solvent Extr. Ion Exch., 31, 401-415 (2013).

9) R.G. Pearson, J. Am. Chem. Soc., 85, 3533-3539 (1963).

10) Y. Morita, J.-P. Glatz, M. Kubota, L. Koch, G. Pagliosa, K. Roemer, A. Nicholl, Solvent Extr. Ion Exch., 14, 385-400 (1996).

11) H. Oigawa, K. Nishihara, S. Nakayama, Y. Morita, Proceedings of Tenth information exchange meeting, Mito, Japan, 123-131 (2008).

12) Y. Sasaki, Y. Kitatsuji, T. Kimura， Chem. Lett., 36, 1394-1395 (2007)

13) Y. Sasaki, Y. Sugo, M. Saeki, Y. Morita, A. Ohashi, Solvent Extr. Res. Dev. Jpn., 18, 69-74 (2011).

14) M. Saeki, Y. Sasaki, A. Nakai, A. Ohashi, D. Banerjee, A.C. Scheinost, and H. Foerstendorf, Inorg. Chem., 51, 5814-5821 (2012).

15) G.E. Boyd and Q.V. Larson, J. Phys. Chem., 64, 988-996 (1960).

16) A. Landgren, J-O. Liljenzin, Solvent Extr. Ion Exch., 17, 1387-1401 (1999).

17) The Chemical Society of Japan, "Kagakubinran $4^{\text {th }}$ edition" Maruzen Ltd., Tokyo (1993). 